\title{
Expression of PAPPA2 in human fetomaternal interface and involvement in trophoblast invasion and migration
}

\author{
H.Y. Wang ${ }^{1,2 *}$, Z. Zhang ${ }^{3 *}$ and S. Yu ${ }^{2}$ \\ ${ }^{1}$ Beijing Shijitan Hospital, Capital Medical University, Beijing, China \\ ${ }^{2}$ Beijing Maternal and Child Health Care Hospital, Capital Medical University, \\ Beijing, China \\ ${ }^{3}$ Anhui Medical University, Hefei, Anhui, China \\ *These authors contributed equally to this study. \\ Corresponding author: S. Yu \\ E-mail: songyu9649@163.com
}

Genet. Mol. Res. 15 (3): gmr.15038075

Received November 17, 2015

Accepted January 18, 2016

Published July 25, 2016

DOI http://dx.doi.org/10.4238/gmr.15038075

Copyright $(\subset 2016$ The Authors. This is an open-access article distributed under the terms of the Creative Commons Attribution ShareAlike (CC BY-SA) 4.0 License.

\begin{abstract}
Pregnancy-associated plasma protein-A 2 (PAPPA2) is a placental-enriched gene that is important for normal human placentation and defects in the gene can cause complications in pregnancy. Yet the exact expression pattern and role of PAPPA2 in the human fetomaternal interface are not clear. In this study, in situ hybridization (ISH) and immunohistochemistry (IHC) were employed to examine the spatial and temporal expression of PAPPA2 in the human fetomaternal interface. IHC results exhibited wide expression of PAPPA2 in the fetomaternal interface, with placental syncytiatrophoblast (STB) and extravillous trophoblast (EVT) showing strong expression and the cytotrophoblast (CTB) showing weak expression of PAPPA2. These results were confirmed by ISH. Quantitative reverse transcription-
\end{abstract}


polymerase chain reaction and western blot showed the elevation of PAPPA2 in first trimester EVT differentiation and term CTB spontaneous syncytialization. PAPPA2-siRNA transfection significantly depressed the invasion and migration ability of a trophoblast cell line (HTR8/SVneo) in a transwell migration and Matrigel invasion model compared to a negative control siRNA $(\mathrm{P}<0.05)$, also revealing that matrix metalloproteinase 9 (MMP9) secretion is downregulated. This was confirmed using a human first trimester placental villi explant culture model. Our results reveal the spatial and temporal expression of PAPPA2 in the human fetomaternal interface and show the positive regulatory role of PAPPA2 in human trophoblast invasion and migration through the secretion of MMP9.

Key words: PAPPA2; Invasion and migration; Trophoblast differentiation; Placenta

\section{INTRODUCTION}

The placenta is a mammalian-specific organ, which is responsible for nutrient and gas exchange between mother and fetus and acts as the barrier protecting the fetus from the maternal immune system (Rawn and Cross, 2008). The human placenta is a typical hemochorial placenta, which is characterized by direct contact between placental trophoblast and maternal blood and immune system (Wildman et al., 2006). Human placenta is highly branched in morphology with the placental villi as its basic functional unit. Placental villi are covered by the outer multi-nuclear syncytiatrophoblast (STB) layer and the underlying mono-nuclear cytotrophoblast (CTB) layer (Rawn and Cross, 2008). The outer layer of arranged STB, which is dynamically maintained through the ejection of syncytial knots and the fusion of underlying $\mathrm{CTB}$, plays an important role in immunotolerance, nutrient exchange, and the production of many important hormones, growth factors, and cytokines (Simpson and MacDonald, 1981). The inner CTB acts as stem-like cells to maintain its ability to self-renew and proliferate the STB. In addition, another major trophoblast lineage, the extravillous trophoblast (EVT), is also differentiated from villous CTB. Under the induction of maternal factors, the CTB firstly degrades the outer enclosed STB and then obtains the ability to be motile (Baczyk et al., 2006). The physiological role of EVT is to gradually invade deep into the maternal decidua and remodel the spiral artery to create a suitable micro-environment for the fetomaternal interface to ensure a successful pregnancy (Ji et al., 2013).

Placentation, constituted by a serial trophoblast linage and morphogenesis, is a strictly regulated genetic process (Hemberger and Cross, 2001). To date, mutant mouse models have allowed partial understanding about the genetic determination for human trophoblast differentiation (Rossant and Cross, 2001). Furthermore, many factors involved in facilitating trophoblast physiological function have also been revealed (Das et al., 2002). Those gene products include proteases (Wang et al., 2009), growth factors and their receptors (Morrish et al., 2007), cytokines (Das et al., 2002), and proteins involved in maintaining cell structures (Cronier et al., 2002). Normal trophoblast lineage and placentation under the regulation of necessary genes, which undergo intrinsic spatial and temporal expression, is the prerequisite for successful pregnancy. Conversely, if the placenta structure is abnormal or trophoblast

Genetics and Molecular Research 15 (3): gmr.15038075 
dysfunction occurs, it is usually accompanied by the abnormal expression of many important genes (Enquobahrie et al., 2008).

Insulin-like growth factor(IGF) I and $\Pi$ are important in regulating cell differentiation, proliferation, and motility (Fowden, 2003). A prerequisite for IGF function is that it needs to be released from the IGF binding protein (IGFBP) (Giudice et al., 2002). Previous work has revealed that pregnancy-associated plasma protein-A (PAPPA) is an important protease for normal placentation through cleaving IGFBP4 to contribute to the release of IGF II (Giudice et al., 2002), which is important for promoting the development of the placenta both for trophoblast proliferation and EVT mobility (Fowden, 2003). Recently, PAPPA2 has been revealed as another important protease which is similar to PAPPA (Farr et al., 2000; Overgaard et al., 2001) and specifically cleaves IGFBP5 (Overgaard et al., 2001; Christians et al., 2006).

The abnormal expression of PAPPA2 in pregnancy complications have been reported (Buimer et al., 2008; Macintire et al., 2014; Munnangi et al., 2014). It has been suggested that PAPPA2 could be an important marker for early onset preeclampsia (PE) (Macintire et al., 2014). Mice models have been established to explore the exact role of PAPPA2 during placentation (Conover et al., 2011) but no obvious placenta defects were reported. Thus, the exact physiological function and direct evidence for the role of PAPPA2 on trophoblast differentiation and placentation is still obscure. Although both humans and mice have hemochorial placentas, the exact structures are very different (Watson and Cross, 2005). The finding that some molecules play a role in mouse trophoblast linage but do not recapitulate their function in human trophoblast differentiation indicates that sometimes mouse models aren't not suitable for exploring the role of genes and proteins in human placentation (Rossant and Cross, 2001). Thus, it is important to determine the exact spatial and temporal expression of PAPPA2 in the fetomaternal interface throughout human gestation. To do this, a human trophoblast cell line model and primary trophoblast model were employed to deduce the physiological role of PAPPA2 during human placentation.

\section{MATERIAL AND METHODS}

\section{Placental tissue collection}

Placental villi, including human first trimester (5-6 weeks), second trimester (20 weeks), and term pregnancy (36-39 weeks), were collected from normal pregnant women undergoing legal abortion or Caesarean section at term in Beijing Shijitan Hospital. Informed written consent for placenta donation were obtained from all pregnant women who donated their placentas. Ethical approval was granted by the Ethics Committee of Beijing Shijitan Hospital. The utilization of samples was guided by standard experimental protocols approved by the Ethics Committee of Beijing Shijitan Hospital. All placental tissues were collected and stored at $4^{\circ} \mathrm{C}$. They were to be transported to the laboratory within $1 \mathrm{~h}$ of surgery and were washed with cold PBS three times before undergoing treatment.

\section{In situ hybridization (ISH)}

Total RNA from fresh placental villi from the first trimester were reverse transcribed to cDNA, which was used as template for amplifying the fragment of transcript to clone

Genetics and Molecular Research 15 (3): gmr.15038075 
the plasmid for probe production. Specific primer pairs used for polymerase chain reaction (PCR) were: (sense) 5'-GTCTGTGACAGTGATTAAGGAGC-3'; (antisense) 5'-CACCAGAGTGATGGGCAGAC-3'. This amplified fragment of $900 \mathrm{bp}$, which localizes at the homologous region of all the known transcript variants of PAPPA2 in GenBank, was cloned into the pGM-T plasmid (Tiangen, Beijing, China) for RNA probe preparation. The sense and antisense RNA probes of PAPPA2 were produced by using the digoxigenin (DIG) RNA labeling kit (SP6/T7, Roche Molecular Biochemicals) in vitro according to the manufacturer instructions. Placental villi from the first trimester and term pregnancy were fixed in $4 \%$ neutral paraformaldehyde (PFA, $\mathrm{pH}$ 7.4) for $1 \mathrm{~h}$ at $4^{\circ} \mathrm{C}$ and dehydrated using gradient glucose ( 5 to $30 \%$ ) within $12 \mathrm{~h}$. After dehydration, placenta blocks were then embedded by Tissue-Tek optimum cutting temperature (OCT) compound (Sakura Finetek, Zoeterwoude, Netherlands). Frozen sections of $6 \mathrm{~mm}$ were sectioned by Leica CM1950 (Leica Microsystems, Wetzlar, Germany). ISH was performed as previously described with small modifications (Chang et al., 2014). DIG-labeled RNA probes of PAPPA2 with a concentration of $2 \mathrm{mg} / \mathrm{mL}$ were used for hybridization at $56^{\circ} \mathrm{C}$ for $16 \mathrm{~h}$. The sections were directly photographed for antisense probe incubation. The sense probe was used as a control for ISH of PAPPA2 and corresponding incubated sections were counter-stained with nuclear fast red solution (Sigma-Aldrich, Germany). Five different normal placental villi of the first trimester and term placenta were utilized to examine the localization of PAPPA2 mRNA in the human fetomaternal interface. Serial sections were incubated with mouse anti-CK7 antibody, a marker used to label the CTB and EVT of human placenta.

\section{Paraffin section and immunohistochemistry (IHC)}

After fixation with $4 \%$ neutral PFA (Sigma-Aldrich) at $4{ }^{\circ} \mathrm{C}$ overnight, placenta blocks were dehydrated with gradient ethanol, embedded in paraffin, and sectioned in $5 \mathrm{~mm}$ slices using a rotary microtome (Leica RM 2135, Leica Microsystems, Bensheim, Germany). IHC was performed using the Streptavidin-Peroxidase Histostain-Plus Kit (Zhongshan Golden Bridge Corp., Beijing, China). After antigen retrieval by citrate solution ( $\mathrm{pH}$ 6.0) and blockage with goat antiserum, the sections were incubated with the primary antibody, rabbit anti-human PAPPA2 (ab59101; Abcam, Cambridge, UK), overnight at $4^{\circ} \mathrm{C}$. Cytokeratine 7 (CK7, clone OV-TL 12/30) was used as the marker for villous CTB and EVT. A serial section incubated with goat anti-rabbit IgG instead of the primary antibody was employed as a negative control. On the following day, the sections were incubated with a peroxidase-conjugated secondary antibody, stained with 3,3'-diaminobenzidine (Zhongshan Golden Bridge Corp.) and then counterstained with hematoxylin. Localization of molecules was determined on sections from five different placentas of the same gestational stage.

\section{Cell culture}

The trophoblast cell line, HTR8/SVneo (Graham et al., 1993), was kindly supplied by Dr. Benjamin K. Tsang (Department of Obstetrics \& Gynecology and Cellular \& Molecular Medicine, University of Ottawa, Canada) and cultured in RPMI 1640 medium (Invitrogen, USA) with $10 \%$ fetal bovine serum (FBS, Thermo Scientific Hyclone, Logan, UT, USA), $100 \mathrm{U} / \mathrm{mL}$ penicillin, and $100 \mathrm{mg} / \mathrm{mL}$ streptomycin. The cells were normally cultured in a $5 \%$ $\mathrm{CO}_{2} / 95 \%$ air incubator at $37^{\circ} \mathrm{C}$.

Genetics and Molecular Research 15 (3): gmr.15038075 


\section{Small-interfering RNA (siRNA) transfection}

SiRNA duplexes specifically targeted to PAPPA2 mRNA were designed and ordered from Genepharma (Shanghai, China). The corresponding sequences of PAPPA2 siRNA were: sense, 5'-GCUUAUCUCGGCAAUCAAATT-3' and antisense, 5'-UUUGAUUGCCGAGAUAAGCTT-3'. A universal negative control siRNA duplex (NC) from Genepharma was utilized as a non-specific control. The reagent employed for transfecting the siRNA into HTR8/SVneo cells was Lipofectamine 2000 (Invitrogen). SiRNA transfection was performed according to the manufacturer instructions with a final concentration of 100 $\mathrm{nM}$. The efficiency of knockdown was examined by semi-quantitative reverse transcription (RT)-PCR and real-time RT-PCR.

\section{Primary CTB isolated from human first trimester placenta and full-term normal placenta}

Placentas were minced into small pieces and digested with $0.25 \%$ trypsin (SH30042.02, Hyclone, Thermo Fisher) and $0.02 \%$ DNase type I (Sigma-Aldrich) at $37^{\circ} \mathrm{C}$ with gentle agitation for three cycles of $15 \mathrm{~min}$ each in order to detach cytotrophoblast cells from the placental villi. The cell suspensions were pooled and loaded on a Percoll gradient (arranged from 70 to $10 \%$ with a $10 \%$ step in gradient), and centrifuged at $1100 \mathrm{~g}$ for $25 \mathrm{~min}$. The cells at Percoll gradient $40-30 \%$ were collected and washed with PBS. The harvested cells were cultured in RPM1640 medium (Invitrogen) supplemented with 10\% FBS, $100 \mathrm{U} / \mathrm{mL}$ penicillin, and $100 \mathrm{mg} / \mathrm{mL}$ streptomycin. Primary term CTBs were also isolated. Briefly, the placenta was digested in DMEM supplemented with 0.125\% trypsin and 0.03\% DNase-I (Sigma-Aldrich) and the CTBs were enriched by Percoll density gradient centrifugation (GE Healthcare, Biosciences AB, Uppsala, Sweden). Full-term CTB were cultured in F12K/DMEM (Invitrogen) medium supplemented with $10 \% \mathrm{FBS}, 100 \mathrm{U} / \mathrm{mL}$ penicillin, and $100 \mathrm{mg} / \mathrm{mL}$ streptomycin.

\section{Real-time RT-PCR}

Total RNA was extracted and purified from fresh human placental villi or cultured cells using TRIzol reagent (Invitrogen). RNA concentration was detected by the NanoDrop 2000 UV-Vis spectrophotometer (Thermo Scientific). Reverse transcription of RNA to cDNA was carried out with PrimeScript ${ }^{\mathrm{TM}}$ RT reagent Kit (Takara, Dalian, China) with $2 \mathrm{mg}$ total RNA. Real-Time PCR was carried out using the SYBR Premix Ex Taq Kit (Takara) and the Real-Time PCR System (ABI PRISM 7500 Real-time PCR System, Applied Biosystems, Foster City, CA, USA). Specific primers used for PCR are listed in Table S1. The data were analyzed by using the $\Delta \mathrm{Ct}$ method and normalized to GAPDH expression (Schmittgen and Livak, 2008).

\section{Matrigel cell invasion and transwell cell migration assays}

Matrigel cell invasion and transwell cell migration assays were performed as previously described (Chang et al., 2014). In summary, HTR8/SVneo cells were transfected with PAPPA2-specific siRNA or the universal negative siRNA (NC). Twenty-four hours after siRNA transfection, $1 \times 10^{5}$ HTR8/SVneo cells in 200 mL RPMI 1640 medium (without FBS)

Genetics and Molecular Research 15 (3): gmr.15038075 
were seeded onto the filter of the transwell chamber $(8.0 \mathrm{~mm}$ pore size for transwell cell migration assay; Corning Life Sciences, Acton, MA, USA) or the transwell which was precoated with Matrigel ( $1 \mathrm{mg} / \mathrm{mL}$ for), and $600 \mathrm{~mL}$ RPMI 1640 medium supplemented with $10 \%$ FBS was added to the bottom chamber. After $24 \mathrm{~h}$, cells on the upper side of the filter were removed and those on the opposite side of transwell membrane were fixed with ice-cold methanol for $10 \mathrm{~min}$ at $4^{\circ} \mathrm{C}$, then stained with hematoxylin and eosin (H\&E) and counted under a light microscope (Olympus IX51, Olympus Corporation). Six fields randomly selected at a magnification of 200X were recorded for statistical analysis. The experiments were carried out in triplicate. The conditional medium from the upper chamber of the transwell of Matrigel invasion assay was collected for gelatin zymography activity assay. The remaining cells transfected with PAPPA2 siRNA or control NC siRNA were used for RNA extraction after transfection for $48 \mathrm{~h}$ to detect the gene silencing efficiency.

\section{Gelatin zymography}

The gelatinolytic activities of matrix metalloproteinase (MMP) 2 and 9 secreted by HTR8/SVneo cells were detected by gelatin zymography. The medium collected from the upper chamber of the transwell was mixed with 4X SDS sample loading buffer (8\% SDS $(\mathrm{w} / \mathrm{v}), 0.04 \%$ bromophenol blue (w/v), $0.25 \mathrm{M}$ Tris-HCl, $\mathrm{pH} 7.6$ ) and incubated at $37^{\circ} \mathrm{C}$ for 1 h. A fixed volume with $15 \mu \mathrm{L}$ each sample was loaded onto and separated by $12 \%$ SDS-PAGE containing $0.5 \mathrm{mg} / \mathrm{mL}$ gelatin (Difco Laboratories, Detroit, MI, USA). After electrophoresis, the gel was gently shaken and incubated with renaturation buffer $(2.5 \%$ Triton X-100, $50 \mathrm{mM}$ Tris- $\mathrm{HCl}, \mathrm{pH} 7.5)$ for $30 \mathrm{~min}$ at room temperature then treated with developing buffer $(50 \mathrm{mM}$ Tris-HCl, pH 7.5, $10 \mathrm{mM} \mathrm{CaCl}_{2}, 1 \mathrm{mM} \mathrm{ZnCl}, 1 \%$ Triton X-100) for $37^{\circ} \mathrm{C}$ overnight. The gel was subsequently stained with $0.5 \%$ Coomassie Brilliant Blue R-250, which was dissolved in $50 \%$ methanol and $10 \%$ acetic acid, for $1 \mathrm{~h}$ at room temperature. After this, the gel was destained in $10 \%$ acetic acid for about $6 \mathrm{~h}$ to visualize the zymogen bands which exhibited as bright white color.

\section{Human first trimester ex vivo extravillous explant culture}

Human placental villi from the first-trimester of pregnancy (5-6 weeks, $\mathrm{n}=12)$ were collected for explant culture according to a previously published method (Baczyk et al., 2009). Anchoring villi were identified by a phase-contrast microscope (Leica S6 D Stereozoom, Leica Microsystems AG) and dissected for explants of 2-5 mm in diameter. The dissected explants were implanted onto the Millicell-CM culture dish inserts (Millipore Corporation, Bedford, MA, USA), which were pre-coated with growth factor-reduced Matrigel (BD Biosciences, Bedford, MA, USA). After the attachment of explants to the Matrigel ( $\sim 6 \mathrm{hrs})$, serum-free DMEM mixed 1:1 with Ham's F-12 (DMEM/F12; Hyclone) was added into the culture dish of the insert chamber. Five hundred milliliters DMEM/F12 medium (containing 10\% FBS, $100 \mathrm{U} / \mathrm{mL}$ penicillin, $100 \mathrm{mg} / \mathrm{mL}$ streptomycin, $2 \mathrm{mM} \mathrm{L}$-glutamine, $100 \mathrm{mg} / \mathrm{mL}$ gentamycin, and $2.5 \mathrm{mg} / \mathrm{mL}$ fungizone) was added to the bottom well. The explants were cultured under $3 \%$ oxygen and $5 \% \mathrm{CO}_{2}$. Followed a previously published protocol (Baczyk et al., 2009), PAPPA2 siRNA (300 nM) was used in DMEM/F12 medium and the medium was replaced with every $24 \mathrm{~h}$. Twenty-four hours later, the explants were observed and recorded using an inverted microscope (Nikon Eclipse Ti, Tokyo, Japan). Only the viable explants presented as

Genetics and Molecular Research 15 (3): gmr.15038075 
EVT outgrowth were observed and maintained for the following experiment. The explants from the same placental villi were assigned into the PAPPA2 siRNA group or the NC group. The outgrowth of explants was monitored and recorded every $24 \mathrm{~h}$ and the explants were cultured for a total of $96 \mathrm{~h}$.

\section{Whole-mount IHC}

To characterize silencing efficiency of the outgrown EVTs from the explanted villi, whole-mount IHC was performed. The explanted villi were fixed using 4\% neutral PFA at $4^{\circ} \mathrm{C}$ for $1 \mathrm{~h}$. After three washes with PBS (containing $1 \%$ Triton), the explanted villi were sequentially incubated with blocking buffer (5\% BSA and goat serum mixed 1:1 with bovine serum albumin) for $1 \mathrm{~h}$, primary antibody against PAPPA 2 at $4^{\circ} \mathrm{C}$ for $24 \mathrm{~h}$, and secondary antibody for $1 \mathrm{~h}$. The procedure was similar to what was described above for IHC. The images were taken using a confocal microscope (Zeiss LSM780, Carl Zeiss AG).

\section{Statistical analysis}

Data are reported as means \pm standard deviation (SD). Statistical analysis was performed by the paired-sample Student $t$-test using the Statistical Package for Social Science (SPSS for Windows package release 10.0, SPSS, Inc., Chicago, USA). $\mathrm{P}<0.05$ was determined to be statistically significant $(\mathrm{P}<0.05 ; \mathrm{P}<0.01)$.

\section{RESULTS}

\section{Expression of PAPPA2 in human placenta}

Two different techniques, ISH and IHC, were employed to determine the exact expression pattern of PAPPA2 in the fetomaternal interface at both mRNA and protein level. PAPPA2 mRNA was highly expressed by villous STB and trophoblast column (TC) of human first trimester placenta (Figure 1A) while it was moderately expressed by the villous CTB and villous stromal cells. Villous CTB and TC of first trimester placenta villi were indicated by the staining with CK7 antibody in the serial sections (Figure 1B). Sections of this stage were incubated with sense probe and positive signal was absent (Figure 1C). The level of PAPPA2 transcript declined in the fetomaternal interface by the third trimester (Figure 1D). A moderate positive signal for PAPPA 2 mRNA was widely distributed in term placental villi while a strong signal was observed in the EVT (Figure 1D), which was indicated by the IHC assay with CK7 antibody in serial sections (Figure 1E). Serial sectioning of term placenta incubated with sense probe was employed as a control for the ISH assay (Figure 1F).

Similar expression patterns for PAPPA2 were also observed at the protein level by the IHC assay (Figure 2). PAPPA2 was abundantly present both in the villous STB and TC of human first trimester placenta (Figure 2A) and moderately expressed by villous CTB and stromal cells (Figure 2A). Similar to what was observed in human first trimester placenta, PAPPA2 in the second trimester ( 20 weeks, Figure 2D) and term placenta (38 week, Figure $2 \mathrm{G}$ ) mainly appeared in the villous STB, EVT in decidua, and villous stromal cells at a moderate level. Meanwhile, the decidua stromal cells were also obviously positive for PAPPA2 antibody staining (Figure 2G). The IHC assay also exhibited that the expression level of PAPPA2 in 
the fetomaternal interface declined along the gestation period. CK7 antibody was employed to label the villous CTB and TC of human first trimester placenta (Figure 2B) and the CTB and EVT of the second (Figure 2E) and third trimester (Figure 2H) placenta. Serial sections of placenta of different gestational age were incubated with rabbit IgG instead of primary antibody and were deemed as the negative control of the IHC assay (Figure 2C, 2F and 2I).

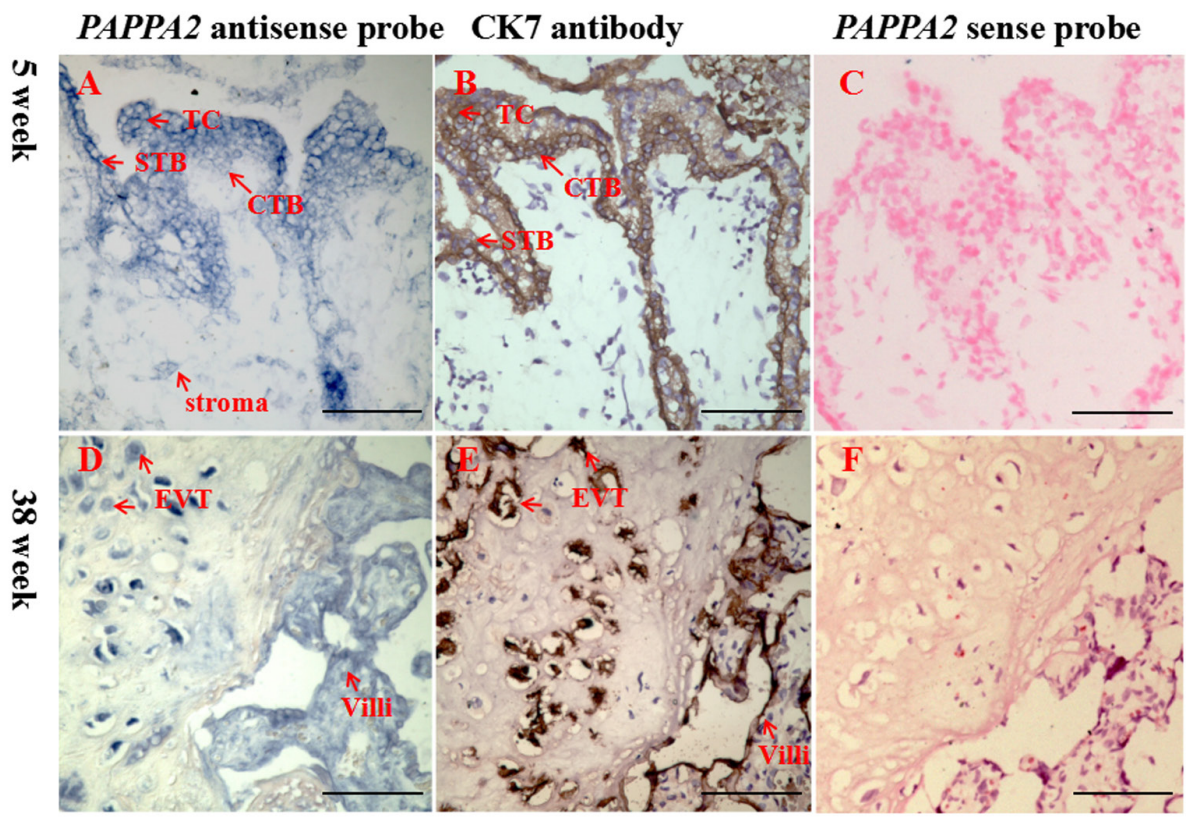

Figure 1. Detecting the localization of PAPPA2 mRNA in human fetomaternal interface by ISH (in situ hybridization). A. Frozen sections were incubated with the antisense probe of PAPPA2. Abundant PAPPA2 mRNA in STB and TC of human first trimester placental villi was observed while the mRNA level in villous CTB and villous stroma cells was moderate. B. CK7 antibody was employed to label the villous CTB and TC of the serial sections of first trimester placenta. C. Sense probe was employed as the negative control and counterstained with nuclear fast red. D. The mRNA level of PAPPA2 was significantly decreased in term placenta villi when compared to first trimester. In frozen section of term placenta, an obvious positive signal for PAPPA2 mRNA was widely distributed at placental villi and EVT in decidua. E. A serial section from term placenta was stained with CK7 antibody, which can label the villous CTB and EVT in decidua. F. No obvious positive signal for the serial section of term placenta incubated with sense probe was observed. ISH, in situ hybridization. CTB, cytotrophoblast. STB, syncytiotrophoblast. TC, trophoblast column. Bar $=100 \mathrm{~mm}$.

\section{Expression of PAPPA2 in the human maternal decidua during the first trimester}

PAPPA2 was strongly expressed in the decidua during the first trimester (Figure 3A). An enlarged view shows that decidua stromal cells and uterine glands were strongly positive for PAPPA2 antibody staining (Figure 3E). The decidua stromal cells and uterine glands were indicated by vimentin antibody (Figure 3C and 3G) and CK7 antibody (Figure 3B and 3F), respectively, in serial section staining. Serial sections incubated with rabbit IgG instead of primary antibody was deemed as negative controls for IHC (Figure 3D and 3H).

Genetics and Molecular Research 15 (3): gmr.15038075 


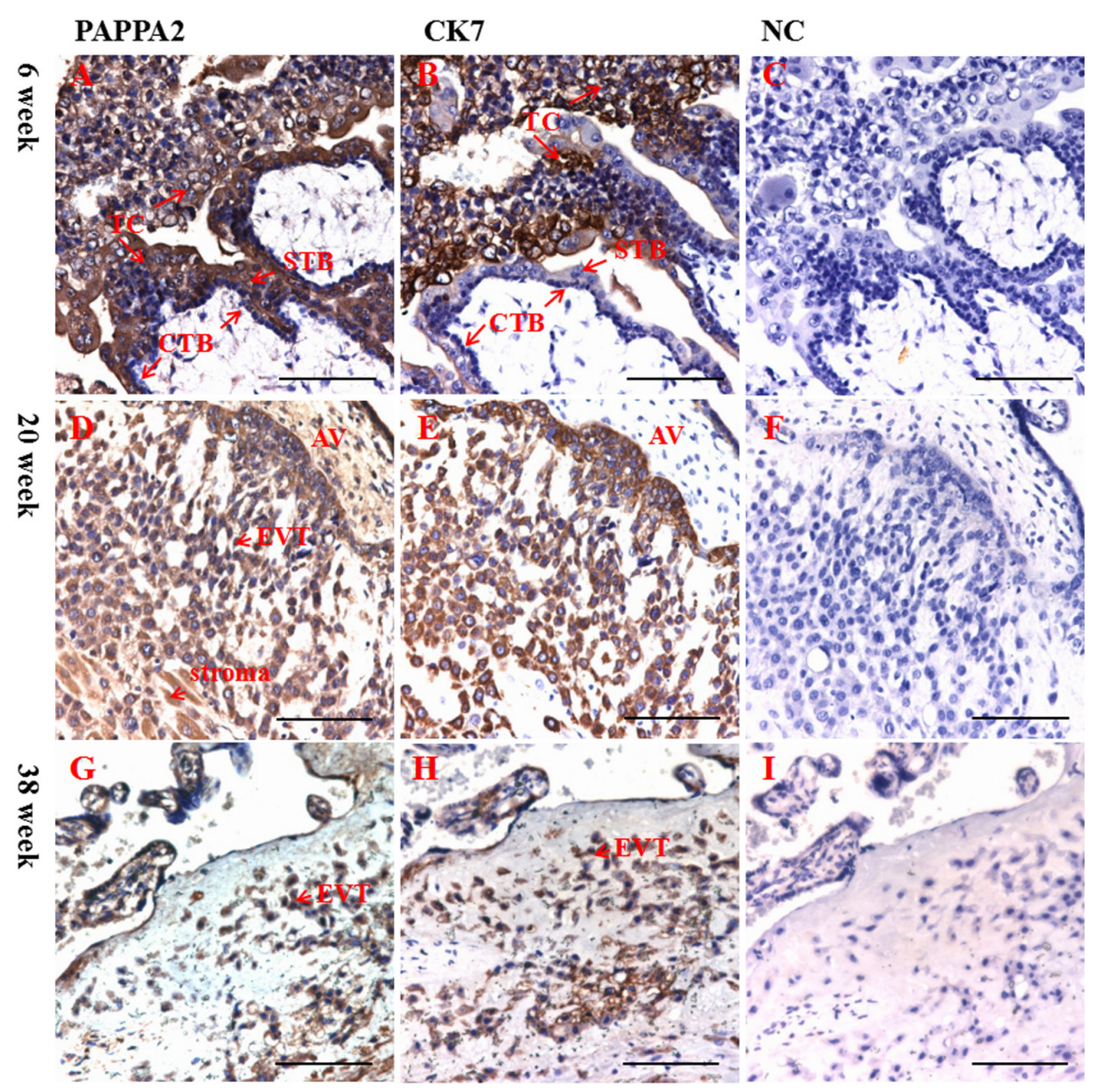

Figure 2. Detection of PAPPA2 expression pattern in the human fetomaternal interface by IHC. A. PAPPA2 was strongly present in villous STB and TC and moderately expressed in villous CTB and stromal cells in the human first trimester tissues. B. CK7 antibody was employed to label villous CTB and TC of human first trimester tissues. C. The serial sections were incubated with rabbit IgG instead of primary antibody as the negative control. D. For second trimester placental villi, PAPPA2 was also mainly observed in villous trophoblast and EVTs, which have invaded the decidua. Similar to the first trimester, the villous stromal cells were also positive for PAPPA2 antibody. E. The serial sections incubated with CK7 antibody was employed to indicate the villous CTB and EVTs. F. No positive signal was observed for the negative control of second trimester. G. Similar to the expression pattern observed in the second trimester placenta, PAPPA2 was mainly expressed by villous trophoblast, villous stroma cells, EVT in decidua, and the uterine stromal cells in term placenta. H. CK7 antibody incubated with the serial section of term placenta showed positive signal in villous CTB and EVT. I. No positive signal was observed in the serial section of term placenta. IHC, immuohistochemistry. CTB, cytotrophoblast. STB, syncytiotrophoblast. TC, trophoblast column. DC, decidua. EVT, extravillous trophoblast. Bar $=100 \mathrm{~mm}$.

\section{PAPPA2 transcription is significantly elevated during both trophoblast EVT differentiation and syncytialization}

EVT development and syncytialization represent the two main differentiation pathways of human trophoblast. Primary CTB from normal human first trimester placenta villi 


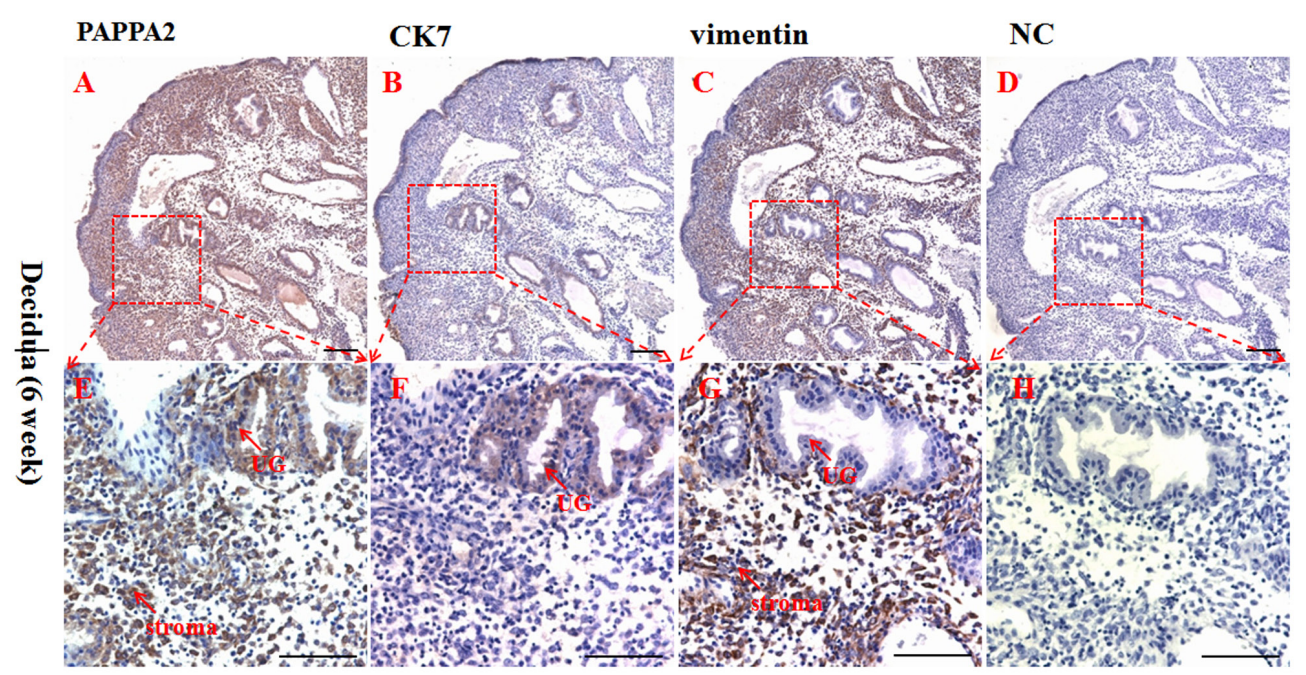

Figure 3. Expression of PAPPA2 by human decidua in first trimester (6 weeks). A. PAPPA2 is strongly expressed in the decidua. CK7 antibody B. and vimentin antibody C. were employed as markers of different decidua components of the first trimester. D. IgG instead of the primary antibody was employed as negative control. E. When observed under further magnification, PAPPA2 was mainly expressed by decidua stromal cells and the glandular epithelium of UG. F. Glandular epithelium of UG was indicated by CK7 antibody in the serial sections. G. Uterine decidua stromal cells were positive for vimentin antibody. UG, uterine gland.

were isolated and induced to EVT in vitro. The significant increase of major histocompatibility complex, class I, G (HLA-G) transcription with a time course pattern indicated the success of this in vitro differentiation model (Figure 4A, $\mathrm{P}<0.01$ ). Along the EVT differentiation process, the significant increase of PAPPA 2 mRNA was exhibited by real-time RT-PCR (Figure $4 \mathrm{~A}, \mathrm{P}<0.05$ ). Isolated CTB from term placenta could spontaneously fuse to form syncytia when cultured in vitro and it is the most ideal model to identify genes involved in the trophoblast syncytialization differentiation pathway. The continual increase in $h C G-\beta$ and syncytin 2 mRNA levels and decrease in $C D H 1$ and GCM1 mRNA levels indicates the progress of trophoblast syncytialization (Figure 4B). The mRNA level of PAPPA2 increased by nearly 300 -fold after three days of culture and differentiation in this study (Figure 4B, P $<$ 0.01). The increase in PAPPA2 during both EVT differentiation and syncytialization was also confirmed by Western blot at the protein level (Figure 4C and 4D).

\section{PAPPA2 is involved in the invasion and migration process of the trophoblast cell line HTR8/SVneo}

The effect of PAPPA2 in trophoblast invasion and migration was measured by Matrigel invasion and transwell migration by employing the trophoblast cell line, HTR8/SVneo, transfected with PAPPA2-specific siRNA (Figure 5A). Results showed that the invasion ability of HTR $8 / \mathrm{SVneo}$ was significantly attenuated by more than $40 \%$ when compared to the negative control (Figure 5A and 5B). The migration efficiency of HTR8/SVneo cells was decreased by $74.5 \%$ after treatment with PAPP2 siRNA when compared to the control treatment (Figure 5A and 5B). The specific PAPPA2 siRNA was efficient to knockdown the expression of PAPPA2, 
A

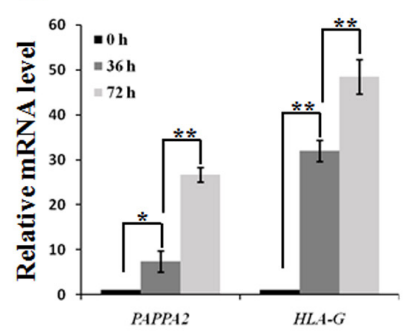

C

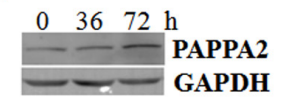

D $\quad 0 \quad 24 \quad 48 \quad 72 \quad \mathrm{~h}$

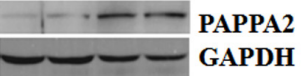

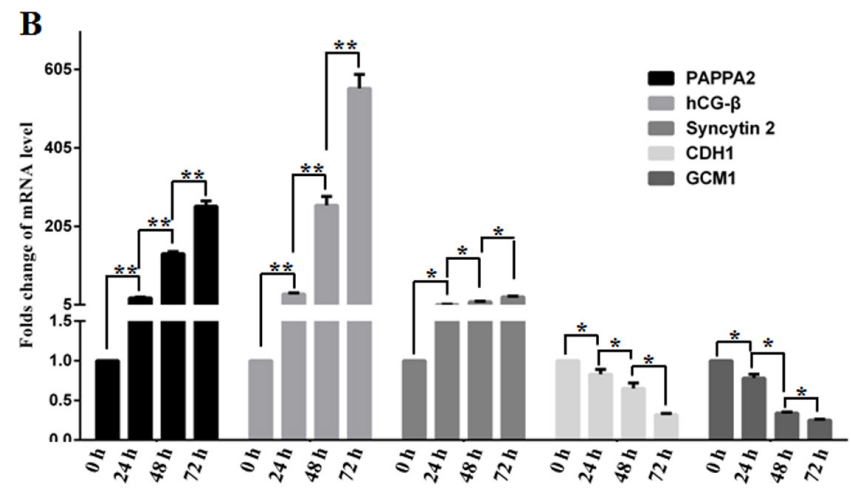

Figure 4. PAPPA2 expression was significantly elevated in both major pathways of human trophoblast differentiation, EVT and syncytialization. A. Transcription of PAPPA2 was significantly increased when EVT was induced in vitro from human first trimester primary EVTs $(\mathrm{P}<0.01)$. B. Transcription level of PAPPA2 was significantly increased in the spontaneous syncytialization process of term primary CTB $(\mathrm{P}<0.01)$. C. D. Corresponding PAPPA2 expression pattern detected the protein level in the EVT differentiation and spontaneous syncytialization process. CTB, cytotrophoblast. EVT, extravillous trophoblast. Bar $=100 \mathrm{~mm} . * \mathrm{P}<0.05$. $* * \mathrm{P}<0.01$.

which was detected by semi-quantitative RT-PCR (Figure 5C) and silencing efficiency was about $75 \%$ as measured by real-time RT-PCR (Figure 5D). We further attempted to understand the mechanism by which PAPPA2 improves the invasion and migration of HTR8/SVneo cells. As illustrated in Figure 5E, the secretion of pro-MMP9 was obviously depressed when HTR8/ SVneo cells were transfected with PAPPA2-specific siRNA.

\section{PAPPA2 is involved in the invasion and migration of EVT in first trimester placenta explant culture}

A primary EVT-inducing model based on human first trimester placental villi explant culture was employed to confirm the role of PAPPA2 in the trophoblast invasion and migration processes. The outgrowth area was obviously different between the placenta explant which was treated with PAPPA2-targeted siRNA and the universal negative siRNA treatment (Figure 6A). Corresponding statistics of the outgrowth area was based on twelve different viable placental explants and showed that when PAPPA2 expression level was efficiently silenced (Figure 6A), the average outgrowth area of EVT was significantly reduced (Figure 6B). 


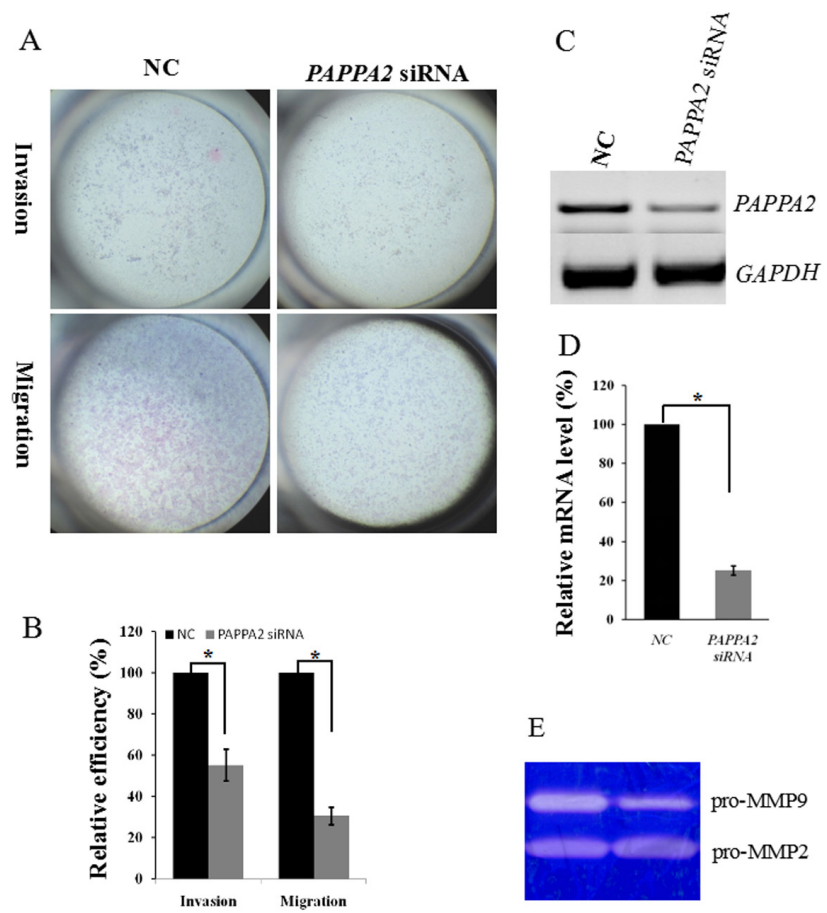

Figure 5. Silencing PAPPA2 significantly impaired the invasion and migration ability of the trophoblast cell line, HTR8/SVneo. A. The invasion and migration ability of HTR8/SVneo was obviously attenuated when transfected with PAPPA2-specific siRNA in the Matrigel invasion and transwell migration model. B. The corresponding quantitation of results based on experiments performed in triplicate. C. Semi-quantitative RT-PCR detection of PAPPA2 mRNA level. D. Quantitative RT-PCR to quantify the knockdown efficiency of PAPPA2 mRNA by specific PAPPA2-siRNA. E. PAPPA2 knockdown can significantly reduce the secretion of pro-MMP9 in HTR8/ SVneo cells, as detected by gelatin zymography. $* \mathrm{P}<0.05$.

$\mathbf{A}$

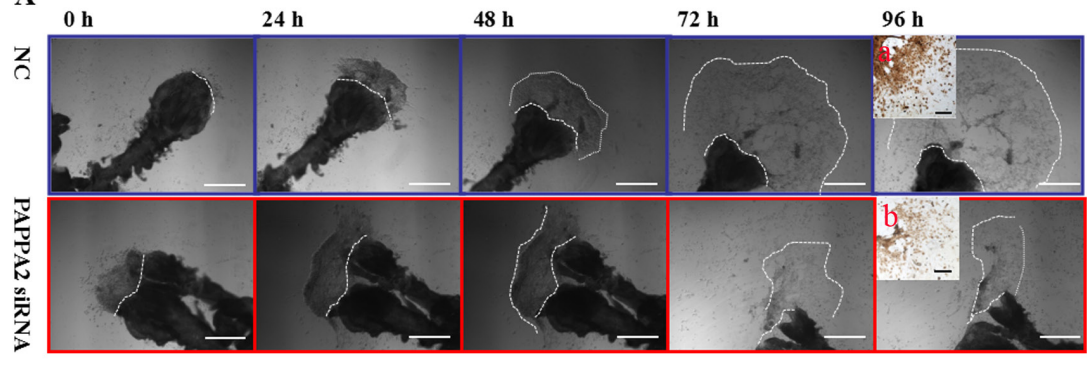

B

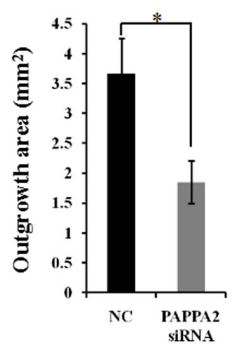

Figure 6. PAPPA2 siRNA transfection impaired the outgrowth area of EVT. A. Primary EVT was induced in human first trimester placental villi explant culture and the outgrowth area was obviously reduced after treatment with PAPPA2 siRNA compared to the universal negative control siRNA (NC). a. and b. Whole mount IHC detecting the PAPPA2 expression level in induced EVT. B. Corresponding quantification of the outgrowth area of the EVT from the NC treatedgroup and PAPPA2 siRNA treated group. EVT outgrowth is outlined by the white dotted line. Bar $=100 \mathrm{~mm}$ in $(\mathrm{A})$.

Genetics and Molecular Research 15 (3): gmr.15038075 


\section{Expression of PAPPA2 mRNA is significantly increased in PE placenta}

PE is characterized by abnormal trophoblast differentiation, insufficient decidua, and spiral artery remodeling due to the dysfunctional EVT pathway. Based on the observation of the abundant expression of PAPPA2 in STB and EVT, the association between PAPPA2 expression in placenta and PE (clinical information listed in Table 1) deserves further attention $(\mathrm{P}<0.05, \mathrm{~N}=8$; Figure 7).

Table 1. Demographic and clinical characteristics of normal and PE patients enrolled in this study.

\begin{tabular}{l|c|c|c}
\hline Characteristics & Control $(\mathrm{N}=8)$ & PE $(\mathrm{N}=8)$ & P value \\
\hline Maternal age (years) & $31.83 \pm 2.48$ & $30.33 \pm 5.16$ & NS \\
\hline Preeclampsia onset (weeks) & None & $24.50 \pm 3.12$ & - \\
\hline Gestational age at delivery (weeks) & $38.23 \pm 0.61$ & $35.35 \pm 3.52$ & NS \\
\hline Primiparae (n) & $100 \%$ & $82.23 \%$ & - \\
\hline Birth weight (g) & $3651.35 \pm 347.23$ & $2390.67 \pm 804.30$ & $<0.05^{*}$ \\
\hline Female fetus (n) & $33.33 \%$ & $33.33 \%$ & - \\
\hline Weight before delivery & $76.00 \pm 4.50$ & $70.38 \pm 9.12$ & NS \\
\hline Prepregnancy weight (kg) & $57.00 \pm 5.09$ & $56.33 \pm 7.24$ & NS \\
\hline Han ethnicity (n) & $100 \%$ & $100 \%$ & - \\
\hline Proteinuria (mg/dL) & $15.36 \pm 3.01$ & $289.00 \pm 152.09$ & $<0.01^{* *}$ \\
\hline Systolic blood pressure (mmHg) & $115.23 \pm 4.32$ & $135 \pm 15.42$ & $<0.05^{*}$ \\
\hline Diastolic blood pressure (mmHg) & $70.33 \pm 2.42$ & $86.39 \pm 4.68$ & $<0.05^{*}$ \\
\hline
\end{tabular}

Values are reported as means $\pm \mathrm{SD}$. Statistical analyses were performed by one-way ANOVA. NS means $\mathrm{P}>0.05$. $* \mathrm{P}<0.05 ; * * \mathrm{P}<0.01$.

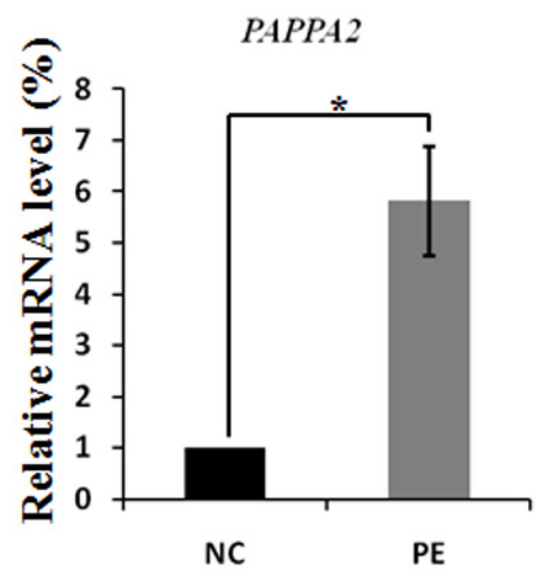

Figure 7. Transcription level of PAPPA2 was increased in placental villi in PE pregnancy. The transcription level was significantly elevated in $\mathrm{PE}$ placenta when compared to paired $\mathrm{NC}$ at the same gestational age $(\mathrm{P}<0.05)$. $\mathrm{NC}$, normal control. $\mathrm{PE}=$ preeclampsia. $\mathrm{N}=8$ (pairs).

\section{DISCUSSION}

In this study, we have confirmed the wide expression of PAPPA2 in the human fetomaternal interface throughout gestation by IHC. PAPPA2 antibody incubation with paraffin sections of different gestational age showed that PAPPA2 is mainly expressed by STB and TC, with weak expression in villous CTB. In addition, uterine gland epithelial and stromal 
cells in both placental villi and maternal decidua were also positive for PAPPA2 antibody at a moderate level. However, Crosley et al. (2014a) showed that PAPPA2 was specifically expressed by villous STB while the expression of its main substrate, IGFBP5, was observed strongly in STB, CTB, and villous stromal cells. It is also the first time that the decreasing expression of PAPPA2 in human placenta along the gestational period was demonstrated. Our observation was different from the report of Wang et al. (2009), who found that the PAPPA2 level was increased in maternal circulation along the gestational period (Wang et al., 2009). Thus, it is reasonable to deduce that the maternal circulatory PAPPA2 in the second and third trimester does not originate from the placenta but actually from the maternal body. The expression pattern of PAPPA2 found in the IHC assay in our study was successfully confirmed by the ISH assay at the mRNA level.

The abundant expression of PAPPA2 in the villous STB and TC led us to be very interested in whether PAPPA2 was involved the trophoblast differentiation pathway, including syncytialization and EVT differentiation. BeWo cells treated with forskolin is extensively utilized as a model to study trophoblast fusion. The expression of PAPPA2 in BeWo cell fusion has also been examined (Wagner et al., 2011). Based on this report, forskolin treatment failed to increase the expression of PAPPA2 in BeWo cells (Wagner et al., 2011). In our study, we isolated primary term CTB from term placenta and cultured them in vitro, allowing them to undergo spontaneous syncytialization. Results showed that the expression of PAPPA2 was significantly elevated both at the mRNA and protein levels. This observation strongly indicated the possibility that PAPPA2 is involved in human trophoblast syncytialization.

Furthermore, the function of PAPPA2 in human trophoblast invasion and migration was examined with specific PAPPA2 siRNA transfection. PAPPA2 enhanced the invasion and migration ability of the trophoblast cell line, HTR8/SVneo. This is the first time that the physiological role of PAPPA 2 in trophoblast cell invasion and migration has been demonstrated directly. The effects of IGF I and II in the invasion and migration process of HTR8/SVneo cells have been reported to be blocked by IGFBP4 and IGFBP5 (Crosley et al., 2014a). Meanwhile, the positive role of IGF I and II on the invasion of DU145 prostate cancer cells was achieved through increasing the enzymatic activity of MMP2 and MMP9 (Saikali et al., 2008). Here, we also showed the positive effect of PAPPA2 on the invasion of HTR8/SVneo cells at least partially through enhancing the secretion of MMP9. The cell line HTR8/SVneo was immortalized from first trimester human placenta and its use to model the character of primary EVT has been contentiously debated because transcriptome assay analysis proved it was different from primary EVT cells (Apps et al., 2011). Thus, primary EVT was necessarily employed to confirm what we found for PAPPA2 in the trophoblast cell line, HTR8/SVneo. Knocking down the expression of PAPPA2 by siRNA also significantly inhibited the outgrowth of EVT in human first trimester placental explant culture. This observation was similar to the result we obtained using the trophoblast cell line, HTR8/SVneo in the transwell model.

Previous work reported that abnormal expression of PAPPA2 was associated with pregnancy complications (Buimer et al., 2008; Crosley et al., 2014a; Macintire et al., 2014; Munnangi et al., 2014). Circulating PAPPA2 was recommend as a valuable marker to screen early onset severe PE (Crosley et al., 2014b; Macintire et al., 2014). PE is typically characterized by insufficient EVT differentiation and inadequate maternal environment remodeling (Lyall et al., 2013) due to the impaired invasion and migration ability of EVT. Here we showed the positive effect of PAPPA2 in regulating trophoblast invasion and migration. Other reports have shown that levels of PAPPA2 are elevated in PE in placenta and maternal serum (Macintire et al.,

Genetics and Molecular Research 15 (3): gmr.15038075 
2014). We also observed the significant elevation of PAPPA2 transcription in term PE placenta by real-time PCR. The possible reason for this phenomenon is that PE leads to inadequate spiral artery remodeling which further contributes to hypoxia in the fetomaternal interface. The hypoxic environment in PE placenta further activates the transcription of PAPPA2 in trophoblast cells (Wagner et al., 2011). The observation that PAPPA2 has the highest expression level in the first trimester but declines in the second and third trimester also coincides with the dynamic change in oxygen concentration in the fetomaternal interface (James et al., 2006). Thus, it appears as if the role of PAPPA2 is to sense the oxygen environment and play a role in EVT by regulating trophoblast function.

In summary, the spatial and temporal expression pattern of PAPPA2 were examined and described in this study. Furthermore, we also found that PAPPA2 positively regulates EVT invasion and migration to modulate the progress of pregnancy.

\section{Conflicts of interest}

The authors declare no conflict of interest.

\section{ACKNOWLEDGMENTS}

Research supported by the International Science and Technology Cooperation Program of China (grant \#2012DFB30130).

\section{REFERENCES}

Apps R, Sharkey A, Gardner L, Male V, et al. (2011). Genome-wide expression profile of first trimester villous and extravillous human trophoblast cells. Placenta 32: 33-43. http://dx.doi.org/10.1016/j.placenta.2010.10.010

Baczyk D, Dunk C, Huppertz B, Maxwell C, et al. (2006). Bi-potential behaviour of cytotrophoblasts in first trimester chorionic villi. Placenta 27: 367-374. http://dx.doi.org/10.1016/j.placenta.2005.03.006

Baczyk D, Drewlo S, Proctor L, Dunk C, et al. (2009). Glial cell missing-1 transcription factor is required for the differentiation of the human trophoblast. Cell Death Differ. 16: 719-727. http://dx.doi.org/10.1038/cdd.2009.1

Buimer M, Keijser R, Jebbink JM, Wehkamp D, et al. (2008). Seven placental transcripts characterize HELLP-syndrome. Placenta 29: 444-453. http://dx.doi.org/10.1016/j.placenta.2008.02.007

Chang WL, Yang Q, Zhang H, Lin HY, et al. (2014). Role of placenta-specific protein 1 in trophoblast invasion and migration. Reproduction 148: 343-352. http://dx.doi.org/10.1530/REP-14-0052

Christians JK, Hoeflich A and Keightley PD (2006). PAPPA2, an enzyme that cleaves an insulin-like growth-factorbinding protein, is a candidate gene for a quantitative trait locus affecting body size in mice. Genetics 173: 15471553. http://dx.doi.org/10.1534/genetics.106.057513

Conover CA, Boldt HB, Bale LK, Clifton KB, et al. (2011). Pregnancy-associated plasma protein-A2 (PAPP-A2): tissue expression and biological consequences of gene knockout in mice. Endocrinology 152: 2837-2844. http://dx.doi. org/10.1210/en.2011-0036

Cronier L, Defamie N, Dupays L, Théveniau-Ruissy M, et al. (2002). Connexin expression and gap junctional intercellular communication in human first trimester trophoblast. Mol. Hum. Reprod. 8: 1005-1013. http://dx.doi.org/10.1093/ $\underline{\text { molehr/8.11.1005 }}$

Crosley EJ, Dunk CE, Beristain AG and Christians JK (2014a). IGFBP-4 and -5 are expressed in first-trimester villi and differentially regulate the migration of HTR-8/SVneo cells. Reprod. Biol. Endocrinol. 12: 123. http://dx.doi. org/10.1186/1477-7827-12-123

Crosley EJ, Durland U, Seethram K, MacRae S, et al. (2014b). First-trimester levels of pregnancy-associated plasma protein A2 (PAPP-A2) in the maternal circulation are elevated in pregnancies that subsequently develop preeclampsia. Reprod. Sci. 21: 754-760. http://dx.doi.org/10.1177/1933719113512532

Das C, Kumar VS, Gupta S and Kumar S (2002). Network of cytokines, integrins and hormones in human trophoblast

Genetics and Molecular Research 15 (3): gmr.15038075 
cells. J. Reprod. Immunol. 53: 257-268. http://dx.doi.org/10.1016/S0165-0378(01)00099-7

Enquobahrie DA, Meller M, Rice K, Psaty BM, et al. (2008). Differential placental gene expression in preeclampsia. Am. J. Obstet. Gynecol. 199: 566.e1-566.e11. http://dx.doi.org/10.1016/j.ajog.2008.04.020

Farr M, Strübe J, Geppert HG, Kocourek A, et al. (2000). Pregnancy-associated plasma protein-E (PAPP-E). Biochim. Biophys. Acta 1493: 356-362. http://dx.doi.org/10.1016/S0167-4781(00)00195-0

Fowden AL (2003). The insulin-like growth factors and feto-placental growth. Placenta 24: 803-812. http://dx.doi. org/10.1016/S0143-4004(03)00080-8

Giudice LC, Conover CA, Bale L, Faessen GH, et al. (2002). Identification and regulation of the IGFBP-4 protease and its physiological inhibitor in human trophoblasts and endometrial stroma: evidence for paracrine regulation of IGF-II bioavailability in the placental bed during human implantation. J. Clin. Endocrinol. Metab. 87: 2359-2366. http:// dx.doi.org/10.1210/jcem.87.5.8448

Graham CH, Hawley TS, Hawley RG, MacDougall JR, et al. (1993). Establishment and characterization of first trimester human trophoblast cells with extended lifespan. Exp. Cell Res. 206: 204-211. http://dx.doi.org/10.1006/ excr.1993.1139

Hemberger M and Cross JC (2001). Genes governing placental development. Trends Endocrinol. Metab. 12: 162-168. http://dx.doi.org/10.1016/S1043-2760(01)00375-7

James JL, Stone PR and Chamley LW (2006). The regulation of trophoblast differentiation by oxygen in the first trimester of pregnancy. Hum. Reprod. Update 12: 137-144. http://dx.doi.org/10.1093/humupd/dmi043

Ji L, Brkić J, Liu M, Fu G, et al. (2013). Placental trophoblast cell differentiation: physiological regulation and pathological relevance to preeclampsia. Mol. Aspects Med. 34: 981-1023. http://dx.doi.org/10.1016/j.mam.2012.12.008

Lyall F, Robson SC and Bulmer JN (2013). Spiral artery remodeling and trophoblast invasion in preeclampsia and fetal growth restriction: relationship to clinical outcome. Hypertension 62: 1046-1054. http://dx.doi.org/10.1161/ HYPERTENSIONAHA.113.01892

Macintire K, Tuohey L, Ye L, Palmer K, et al. (2014). PAPPA2 is increased in severe early onset pre-eclampsia and upregulated with hypoxia. Reprod. Fertil. Dev. 26: 351-357. http://dx.doi.org/10.1071/RD12384

Morrish DW, Kudo Y, Caniggia I, Cross J, et al. (2007). Growth factors and trophoblast differentiation--workshop report. Placenta 28 (Suppl A): S121-S124. http://dx.doi.org/10.1016/j.placenta.2007.02.001

Munnangi S, Gross SJ, Madankumar R, Salcedo G, et al. (2014). Pregnancy associated plasma protein-A2: a novel biomarker for Down syndrome. Placenta 35: 900-906. http://dx.doi.org/10.1016/j.placenta.2014.08.001

Overgaard MT, Boldt HB, Laursen LS, Sottrup-Jensen L, et al. (2001). Pregnancy-associated plasma protein-A2 (PAPP-A2), a novel insulin-like growth factor-binding protein-5 proteinase. J. Biol. Chem. 276: 21849-21853. http:// dx.doi.org/10.1074/jbc.M102191200

Rawn SM and Cross JC (2008). The evolution, regulation, and function of placenta-specific genes. Annu. Rev. Cell Dev. Biol. 24: 159-181. http://dx.doi.org/10.1146/annurev.cellbio.24.110707.175418

Rossant J and Cross JC (2001). Placental development: lessons from mouse mutants. Nat. Rev. Genet. 2: 538-548. http:// dx.doi.org/10.1038/35080570

Saikali Z, Setya H, Singh G and Persad S (2008). Role of IGF-1/IGF-1R in regulation of invasion in DU145 prostate cancer cells. Cancer Cell Int. 8: 10. http://dx.doi.org/10.1186/1475-2867-8-10

Schmittgen TD and Livak KJ (2008). Analyzing real-time PCR data by the comparative C(T) method. Nat. Protoc. 3 : 1101-1108. http://dx.doi.org/10.1038/nprot.2008.73

Simpson ER and MacDonald PC (1981). Endocrine physiology of the placenta. Annu. Rev. Physiol. 43: 163-188. http:// dx.doi.org/10.1146/annurev.ph.43.030181.001115

Wagner PK, Otomo A and Christians JK (2011). Regulation of pregnancy-associated plasma protein A2 (PAPPA2) in a human placental trophoblast cell line (BeWo). Reprod. Biol. Endocrinol. 9: 48. http://dx.doi.org/10.1186/1477$\underline{7827-9-48}$

Wang J, Qiu Q, Haider M, Bell M, et al. (2009). Expression of pregnancy-associated plasma protein A2 during pregnancy in human and mouse. J. Endocrinol. 202: 337-345. http://dx.doi.org/10.1677/JOE-09-0136

Watson ED and Cross JC (2005). Development of structures and transport functions in the mouse placenta. Physiology 20 : 180-193. http://dx.doi.org/10.1152/physiol.00001.2005

Wildman DE, Chen C, Erez O, Grossman LI, et al. (2006). Evolution of the mammalian placenta revealed by phylogenetic analysis. Proc. Natl. Acad. Sci. USA 103: 3203-3208. http://dx.doi.org/10.1073/pnas.0511344103

\section{Supplementary material}

Table S1. Primer pairs for real-time PCR.

Genetics and Molecular Research 15 (3): gmr.15038075 